\title{
Psicooncología
}

ISSN: 1696-7240

\section{Propiedades psicométricas del Cuestionario General de Salud de Goldberg -GHQ-28- en cuidadores primarios informales de pacientes con cáncer}

\author{
Oscar Galindo Vázquez 1,*; Abelardo Meneses García²; Ángel Herrera Gómez³ Paula Cabrera \\ Galeana $^{4}$; Laura Suchil Bernal ${ }^{5}$; Liliana Rivera Fong ${ }^{6}$; José Luis Aguilar Ponce ${ }^{7}$
}

Recibido: 8 de febrero de 2017 / Aceptado: 14 de abril de 2017

Resumen. Objetivo. Determinar las propiedades psicométricas del Cuestionario de Salud General de Goldberg -GHQ-28-, en una muestra de cuidadores primarios informales mexicanos de pacientes con cáncer. Método. Participaron 276 cuidadores de los cuales 205 fueron mujeres $(74,3 \%)$ y 71 hombres (25,7\%). Los participantes contestaron concurrentemente el GHQ-28 y el Inventario de Depresión de Beck BDI. Resultados. La consistencia interna de la escala global mostró un índice satisfactorio $(\alpha=0,91)$. Las alfas de Cronbach de las sub-escalas tuvieron un valor de $\alpha=0,86$, a $\alpha=0,76$ que explicaron el $53,42 \%$ de la varianza. La validez concurrente con el BDI mostró resultados significativos ( $\mathrm{r}$ de Pearson de 0,72 a $0,427, p<0,05$ ). Conclusiones. La relevancia de los resultados obtenidos radica en que se trata de una población que puede llegar a presentar afectaciones en la salud en general a lo largo del proceso de cuidado de los pacientes oncológicos.

Palabras clave: Cáncer, Cuidadores primarios, Pacientes, Población mexicana, Salud general.

\section{[en] Psychometric properties of Goldberg's General Health Questionnaire -GHQ-28- in informal primary caregivers of cancer patients}

\begin{abstract}
Objective. To determine the psychometric properties of Goldberg's General Health Questionnaire -GHQ-28- in a sample of Mexican primary caregivers of cancer patients. Method: Two hundred and seventy six caregivers participated in this protocol, 205 of which were women $(74.3 \%)$ and 71 were men (25.7\%). Participants answered the GHQ-28 and the Beck Depression Inventory (BDI) concurrently. Results. The internal consistency of the overall scale showed a satisfactory index $(\alpha=0.91)$.
\end{abstract}

1 Servicio de Psico-oncología, Instituto Nacional de Cancerología, Mexico. Facultad de Psicología, Universidad Nacional Autónoma de México.

E-mail: psigalindo@yahoo.com.mx

2 Dirección General, Instituto Nacional de Cancerología, México.

E-mail: amenesesg@incan.edu.mx

3 Dirección General Adjunta Médica, Instituto Nacional de Cancerología, México.

E-mail: aherreragc@incan.edu.mx

4 Departamento de Tumores Mamarios, Instituto Nacional de Cancerología, México.

E-mail: dra.paulacabrera@gmail.com

5 Vinculación Institucional, Instituto Nacional de Cancerología, México.

E-mail: vinculacion.incan@gmail.com

6 Facultad de Psicología, Universidad Nacional Autónoma de México, México.

E-mail: 1meylenf@hotmail.com

7 Subdirección de Medicina Interna, Instituto Nacional de Cancerología, México. E-mail: oncoincan@hotmail.com

* Dirección de correspondencia: Dr. Oscar Galindo Vázquez . Servicio de Psicooncología, Instituto Nacional de Cancerología. Av. San Fernando No. 22. Col. Sección XVI. C.P. 14080 Deleg. Tlalpan, México, D.F. E-mail: psigalindo@yahoo.com.mx 
The Cronbach alphas of the subscale had a value of $\alpha=.86, \alpha=.76$, explaining the $53.42 \%$ of the variance. The correlation validity by BDI showed significant outcomes (Pearson $\mathrm{r}=0.723-0.427, p<0.05$ ).

Conclusions. The relevance of the outcomes is that this is a population that can show damages in the overall health throughout the process of care for cancer patients.

Keywords: Cancer, primary caregivers, patients, Mexican Population, General Health.

Sumario. 1. Introducción 2. Método 2.1. Muestra 3. Instrumentos 3.1. Cuestionario de Salud General de Goldberg-GHQ-28-3.2. Inventario de Depresión de Beck - BDI 4. Análisis estadísticos 5. Resultados 5.1. Estructura factorial 5.2. Consistencia interna 5.3. Validez externa 6. Discusión 6.1. Implicaciones para la práctica y la investigación 6.2. Limitaciones del estudio 7. Referencias bibliográficas

Cómo citar: Galindo O, Meneses A, Herrera Á, Cabrera P, Suchil L, Rivera L, et al. Propiedades psicométricas del Cuestionario General de salud de Goldberg -GHQ-28- en cuidadores primarios informales de pacientes con cáncer. Psicooncologia 2017; 14:71-82. DOI: 10.5209/PSIC.55812

\section{Introducción}

En el mundo cada día hay mayor número de personas que enferman de cáncer y, a nivel mundial, se ha convertido en la primer causa de muerte con 8,2 millones de fallecimientos solo en el 2012 (Organización Mundial de la Salud, OMS, 2015) ${ }^{(1)}$.

Sobre ello se debe considerar que el cáncer es actualmente una enfermedad crónica y degenerativa que en algunos países se sigue diagnosticando en etapas avanzadas; situación que incrementa el riesgo de que el paciente pueda ir perdiendo independencia y generando discapacidad prematura que la lleva a tener cada vez más la necesidad de ser asistido ${ }^{(2-4)}$ es decir de requerir de cuidados a largo plazo que de acuerdo con la Organización Mundial de la Salud (2002) ${ }^{(2)}$ se definen como todas aquellas actividades realizadas por un largo periodo de tiempo sobre una persona que no es totalmente capaz de cuidarse por sí misma; y que forman parte de las tareas esenciales para el tratamiento óptimo del paciente oncológico ${ }^{(5)}$.

Generalmente estas acciones son desempeñadas en la mayoría de los casos, por cuidadores informales (familiares y amigos) quienes se convierten en un cuidador primario, la OMS (1999) ${ }^{(6)}$ lo define como: "la persona del entorno de un paciente que asume voluntariamente el papel de responsable del mismo en un amplio sentido; este individuo está dispuesto a tomar decisiones por y para el paciente y a cubrir las necesidades básicas del mismo, ya sea de manera directa o indirecta".

Se ha descrito un perfil del cuidador en el que predominantemente es desempeñado por mujeres, en edad productiva, con un nivel educativo bajo ${ }^{(7,8)}$. Este rol no es una tarea fácil, por el contrario, frecuentemente implica detener momentáneamente planes y actividades cotidianas para ocuparse "completamente" del enfermo ${ }^{(9)}$.

Los cuidadores primarios comúnmente se comprometen en tareas complejas de cuidado entre las que se encuentran: (a) labores de higiene, (b) manejo de síntomas, (c) administración de medicamentos, (d) transporte a los centros hospitalarios, (e) coordinación de citas médicas, (f) atención emocional y (g) comunicación con el equipo de salud ${ }^{(7,10-11)}$.

Como parte del impacto en la vida de los cuidadores, se ha documentado que aquellos que asisten a un paciente con cáncer tienen restricciones significativas 
en sus actividades sociales, recreativas y financieras, así como carga física ${ }^{(13,14)}$; de manera específica se ha observado que los cuidadores primarios consideran que tienen una salud mala con especial afectación en las esferas de energía, sueño, relaciones sociales y emocionales; además de que tienen mayor riesgo de presentar cefalea, dolor de espalda, cansancio/fatiga, y apatía; a nivel psicológico con sintomatología de ansiedad, depresión, mayor disfuncionalidad familiar y sensación de apoyo insuficiente ${ }^{(15-18)}$.

Esta sintomatología presenta una tendencia a incrementarse de acuerdo a la evolución del paciente y a que en los cuidadores primarios es poco frecuente la expresión de sus necesidades; ya que existe una percepción de no desear molestar al personal médico que está ocupado atendiendo a su paciente ${ }^{(19)}$; además de que, a pesar de ser una figura central en el tratamiento del paciente, su evaluación especializada es poco frecuente; a manera de ejemplo se ha descrito que por cada siete instrumentos que miden calidad de vida en pacientes se desarrollan uno que evalúe la calidad de vida de los cuidadores ${ }^{(20)}$.

En este contexto, el Cuestionario de Salud General de Goldberg - GHQ (Goldberg, 1978)(21), es un instrumento diseñado con el fin de evaluar el grado de salud general ${ }^{(22)}$; sobre el cual se han elaborado versiones de 30, 28 y 12 ítems. El GHQ ha sido adaptado y validado en diversas poblaciones, mostrando un alfa de Cronbach entre 0,89 y $0,75^{(22-29)}$ y se ha empleado en estudios epidemiológicos, de screening, con la intención de evaluar la salud mental a nivel de población general, jóvenes estudiantes, ancianos, mujeres embarazadas y puérperas, familiares y cuidadores de enfermos crónicos ${ }^{(30)}$.

Sin embargo, en México no existe una Versión del Cuestionario General de Salud de Goldberg para cuidadores primarios informales de pacientes con cáncer. Por lo cual el objetivo de este estudio fue obtener las propiedades psicométricas del GHQ-28.

\section{Método}

Se incluyeron 276 cuidadores primarios de pacientes oncológicos de ambos sexos entre 43,4 $\pm 12,8$ años (Ver tabla 1 para una descripción de la muestra). La obtención de la muestra fue por disponibilidad en el Instituto Nacional de Cancerología (INCan) de México.

Tabla 1. Descripción de la muestra.

\begin{tabular}{lrrrrr}
\hline & f & $\mathbf{\%}$ & f & \% \\
\hline N & 276 & 100 & Estado civil & & \\
Edad (Rango) 16-77 & & & Soltero(a) & 56 & 20,3 \\
& & & Casado o Unión libre & 179 & 64,9 \\
Sexo & & & Divorciado(a) & 30 & 10,9 \\
Masculino & 71 & 25,7 & Viudo & 11 & 4,0 \\
Femenino & 205 & 74,3 & & & \\
Escolaridad & & & Parentesco & & \\
& & & Esposo(a) & 174 & 63,1
\end{tabular}




\begin{tabular}{lrrlrr} 
Ninguno & 9 & 3,3 & Hijo(a) & 78 & 27,9 \\
Primaria & 64 & 23,2 & Otros & 24 & 0,9 \\
Secundaria & 101 & 36,6 & & & \\
Bachillerato & 66 & 23,9 & Dx. del paciente & & \\
Licenciatura & 25 & 9,1 & Mama & 78 & 28,3 \\
Posgrado & 11 & 4,0 & Cabeza y cuello & 21 & 7,6 \\
& & & Urogenitales & 14 & 7,3 \\
& & & Ginecológicos & 37 & 10,9 \\
& & Hematológico & 15 & 14,5 \\
& & Otros & 111 & 68,6 \\
\hline
\end{tabular}

\subsection{Muestra}

La muestra de participantes se obtuvo durante las consultas de los Servicios de Oncología Médica, Cirugía y Psico-oncología tanto en consulta externa y hospitalización. El proyecto fue aprobado por los Comités Científico y de Ética del Instituto Nacional de Cancerología. Cada uno de los participantes aceptó participar en el presente estudio mediante la comprensión y firma del consentimiento informado de investigación.

Los participantes fueron seleccionados a partir de los siguientes criterios de inclusión: (a) familiar, conocido o amigo que de forma verbal manifiesta ser el principal proveedor de las actividades de cuidado, (b) cuidadores de pacientes con cualquier diagnóstico oncológico, estadio y/o tratamiento, (c) saber leer y escribir, (d) no recibir ninguna remuneración económica por los servicios que ofrece al paciente y (e) sin formación previa, en actividades de cuidado a pacientes.

Como criterios de exclusión se consideró: (a) cuidadores con deficiencias cognitivas y (b) con problemas auditivos y visuales severos.

Los participantes además contestaron una cédula de identificación que recabaron los datos sociodemográficos que incluyeron edad, sexo, parentesco, escolaridad y diagnóstico del paciente.

\section{Instrumentos}

\subsection{Cuestionario de Salud General de Goldberg - GHQ-28 -}

El Cuestionario General de Salud de Goldberg (1978) ${ }^{(21)}$ validada para población mexicana general por Romero y Medina-Mora $(1987)^{(27)}$ es un instrumento dirigido a diferenciar probables pacientes psiquiátricos de aquellos considerados como potencialmente normales. Está originalmente diseñado para identificar trastornos mentales no psicóticos en contextos de práctica médica general. El GHQ-28 consta de cuatro subescalas de siete ítems cada una. La escala A refiere síntomas somáticos; la $\mathrm{B}$, ansiedad e insomnio; la $\mathrm{C}$, disfunción social y la $\mathrm{O}$, depresión severa. Ambas versiones mantienen un buen grado de concordancia, siendo los coeficientes de validez y fiabilidad del GHQ-28 semejantes a los de la versión de 60 ítems. La versión mexicana ha mostrado adecuada consistencia interna $(0,90)$ y validez divergente moderada $\mathrm{r}=0,60$. 


\subsection{Inventario de Depresión de Beck - BDI}

El Inventario de Depresión de Beck -BDI- (Beck, Ward, Mendelson, Mock, \& Erbaungh, 1961) $)^{(31)}$ fue estandarizado para población mexicana por Jurado et al. $(1998)^{(32)}$ y diseñado para evaluar la intensidad de sintomatología depresiva. Es autoaplicable y consta de 21 reactivos, con cuatro opciones de respuesta que describen el espectro de severidad de la categoría sintomática y conductual. Consta de tres factores que evalúan: 1) ánimo, 2) pesimismo, 3) sensación de fracaso, 4) insatisfacción, 5) sentimientos de culpa, 6) sensación de castigo, 7) autoaceptación, 8) autoacusación, 9) ideación suicida, 10) llanto, 11) irritabilidad, 12) aislamiento, 13) indecisión, 14) imagen corporal, 15) rendimiento laboral, 16) trastornos del sueño, 17) fatigabilidad, 18) apetito, 19) pérdida de peso, 20) preocupación somática y 21) pérdida de libido. En población mexicana muestra un alfa de Cronbach $=0,87$. Así como una validez concurrente entre el BDI y la Escala de Zung, de $r=0,70, p<0,01$.

\section{Análisis estadísticos}

Para el análisis de los datos se usó el paquete estadístico SPSS versión 14.0 para Windows. Se obtuvieron los estadísticos de frecuencias de cada reactivo para conocer su distribución en las opciones de respuestas; posteriormente se determinaron los grupos extremos (cuartil 25 y 75) basados en la calificación obtenida por los participantes y con cada reactivo se llevó a cabo una prueba t de Student para muestras independientes para identificar la propiedad de discriminación de cada reactivo, aquellos que tuvieron una $\mathrm{p}>0,05$ fueron eliminados de las siguientes pruebas.

Posteriormente se realizaron análisis de tablas cruzadas para conocer la correlación entre ítems y determinar el tipo de análisis factorial; Se determinó la estructura factorial por medio de un análisis factorial de componentes principales con rotación Varimax. Los criterios utilizados para el análisis factorial fueron: (a) cargas factoriales $\geq 0,40$; (b) factores con por lo menos tres ítems, y (c) coeficiente de consistencia interna por cada factor alfa de Cronbach $\geq 0,60$. Se determinó la validez concurrente entre el GHQ-28 y el BDI mediante el coeficiente de correlación de Pearson.

\section{Resultados}

\subsection{Estructura factorial}

Se obtuvo en la prueba de esfericidad de Bartlett una $(\mathrm{p}=0,001)$ y en la prueba de Kaiser-Meyer-Olkin, de 0,88. El análisis factorial con 28 reactivos arrojó un modelo con cuatro factores con valores arriba de 1,00. El ítem siete " ¿Ha tenido temperatura corporal alta o escalofríos?" fue eliminado debido a que mostró una carga factorial $\leq$ a 0,40 (ver tabla 2 ). 
Tabla 2. Análisis factorial GHQ-28.

\begin{tabular}{|c|c|c|c|}
\hline $\begin{array}{l}\text { Escala Global } \alpha=0,91 \\
\text { Varianza Explicada }=53,42 \%\end{array}$ & $\begin{array}{l}\text { Carga } \\
\text { factorial }\end{array}$ & $\begin{array}{l}\text { Varianza } \\
\text { explicada }\end{array}$ & $\begin{array}{l}\text { Alfa de } \\
\text { Cronbach }\end{array}$ \\
\hline Factor 1. Síntomas somáticos & & $30,99 \%$ & $\alpha=, 86$ \\
\hline 1. Se ha sentido perfectamente bien de salud y en plena forma. & 643 & & \\
\hline $\begin{array}{l}\text { 2. Ha tenido la sensación de que necesitaba algún tipo de } \\
\text { vitaminas. }\end{array}$ & 654 & & \\
\hline 3. Se ha sentido agotado(a) y sin fuerzas para nada. & 687 & & \\
\hline 4. Ha tenido la sensación de que estaba enfermo(a). &, 780 & & \\
\hline 8. Sus preocupaciones le han hecho perder mucho sueño. &, 560 & & \\
\hline 9. Ha tenido dificultades para dormir toda la noche. & 636 & & \\
\hline 10. Se ha notado constantemente agobiado(a) y en tensión. & 619 & & \\
\hline 16. Le cuesta más tiempo hacer las cosas. & ,401 & & \\
\hline Factor 2. Ansiedad y dolor de cabeza & & $9,84 \%$ & $\alpha=, 86$ \\
\hline 5. Ha padecido dolores de cabeza. & ,441 & & \\
\hline 6. Ha tenido sensación de opresión en la cabeza o de que la & & & \\
\hline cabeza le va a estallar. &, 555 & & \\
\hline $\begin{array}{l}\text { 11. Se ha sentido con los nervios a flor de piel y } \\
\text { malhumorado(a). }\end{array}$ & 661 & & \\
\hline 12. Se ha asustado o ha tenido pánico sin motivo. & ,642 & & \\
\hline 13. Ha tenido sensación de que todo se le viene encima. & ,723 & & \\
\hline 14. Se ha sentido nervioso y "a punto de explotar" & & & \\
\hline constantemente. & ,803 & & \\
\hline 26. Ha notado que a veces no puede hacer nada porque tiene los & & & \\
\hline nervios fuera de control. & 644 & & \\
\hline Factor 3. Funcionamiento social & & $7,70 \%$ & $\alpha=, 76$ \\
\hline 15. Se las ha arreglado para mantenerse ocupado y activo. &, 565 & & \\
\hline $\begin{array}{l}\text { 17. Ha tenido la impresión, en conjunto, de que está haciendo } \\
\text { bien las cosas. }\end{array}$ & 664 & & \\
\hline $\begin{array}{l}\text { 18. Se ha sentido satisfecho(a) con su manera de hacer las } \\
\text { cosas. }\end{array}$ &, 770 & & \\
\hline 19. Ha sentido que está desempeñando un papel útil en la vida. & ,668 & & \\
\hline 20. Se ha sentido capaz de tomar decisiones. &, 710 & & \\
\hline $\begin{array}{l}\text { 21. Ha sido capaz de disfrutar de sus actividades normales de } \\
\text { cada día. }\end{array}$ &, 517 & & \\
\hline Factor 4. Depresión e ideación suicida. & & $4,87 \%$ & $\alpha=, 80$ \\
\hline 22. Ha pensado que usted es una persona que no vale para nada. &, 573 & & \\
\hline 23. Ha estado viviendo la vida totalmente sin esperanza. & 622 & & \\
\hline $\begin{array}{l}\text { 24. Ha tenido el sentimiento de que la vida no merece la pena } \\
\text { vivirse. }\end{array}$ &, 847 & & \\
\hline 25. Ha pensado en la posibilidad de "quitarse de en medio" & ,741 & & \\
\hline 27. Ha notado que desea estar muerto y lejos de todo. &, 804 & & \\
\hline $\begin{array}{l}\text { 28. Ha notado que la idea de quitarse la vida le viene } \\
\text { repentinamente a la cabeza }\end{array}$ & 611 & & \\
\hline
\end{tabular}




\subsection{Consistencia interna}

La consistencia interna del GHQ-28 mostró un índice adecuado $(\alpha=0,91)$. Las alfas de Cronbach por subescala tuvieron los siguientes valores: Síntomas somáticos $\alpha=0,86$; ansiedad y dolor de cabeza $\alpha=0,86$; funcionamiento social $\alpha=0,76$ y depresión e ideación suicida $\alpha=0,80$ que explican el $53,42 \%$ de la varianza.

\subsection{Validez externa}

La validez por medio de la correlación entre el GHQ-28 y el BDI, identificaron asociaciones positivas, estadísticamente significativas y teóricamente relevantes de moderadas a altas ( $\mathrm{r}$ de Pearson de 0,723 a $0,427, p<0,05$ ). (Ver tabla 3 ).

Tabla 3. Correlaciones entre el GHQ-28 y BDI.

\begin{tabular}{lccccc}
\hline & 1 & 2 & 3 & 4 & 5 \\
\hline 1. GHQ-28 & & & & & \\
2. F1. Síntomas somáticos &, $888^{*}$ & & & & \\
3. F2. Ansiedad &, $873^{*}$ &, $721^{*}$ & & & \\
4. F3. Disfunción social &, $671^{*}$ &, $479^{*}$ &, $401^{*}$ & & \\
5. F4. Depresión e ideación suicida. &, $534^{*}$ &, $292^{*}$ &, $338^{*}$ &, $242^{*}$ & \\
6. BDI Beck &, $723^{*}$ &, $604^{*}$ &, $672^{*}$ &, $432^{*}$ &, $427^{*}$ \\
\hline
\end{tabular}

\section{Discusión}

El objetivo de este trabajo fue obtener las propiedades psicométricas del GHQ-28 en cuidadores primarios informales mexicanos de pacientes con cáncer; ya que se ha documentado el impacto en la salud de esta población ${ }^{(33,34)}$ y que dicho efecto se incrementa en relación a la evolución del paciente oncológico ${ }^{(19)}$. Por lo que resulta indispensable contar con instrumentos válidos y confiables para evaluar la salud general de los cuidadores que nos permitan identificar objetiva y oportunamente áreas de oportunidad para el desarrollo de intervenciones específicas. En este sentido el Cuestionario de Salud General de Goldberg - GHQ - (Goldberg, 1978) ${ }^{(21)}$ ha mostrado utilidad en diferentes estudios de validación efectuados ${ }^{(23-27)}$.

De acuerdo con los resultados mostrados en este estudio hay un importante predominio de mujeres que fungen como cuidadores primarios $(74 \%)$, lo que coincide con la literatura internacional que reportan que entre del $62 \%$ al $83 \%$ de los cuidadores son de este género ${ }^{(2,19)}$ y que pudiera tener implicaciones ya que los cuidadores de sexo femenino tienden a invertir más tiempo y dar una atención más personal que los de sexo masculino ${ }^{(35)}$.

En relación a la edad, se obtuvo una media de 43 años; este dato es de vital importancia, ya que se ha documentado que cuidadoras más jóvenes muestran niveles de sobrecarga más altas que las de mayor edad, probablemente debido a que perciben un mayor costo de oportunidad asociado a cuidar ${ }^{(36)}$. 
Por otro lado, el nivel de estudios fue de secundaria o menos en el $62 \%$, se ha observado que esta variable tiene una correlación negativa con la carga del cuidador, es decir que a menor nivel de estudios, se experimenta mayor carga y menor salud física que aquellos con mayor nivel educativo ${ }^{(37,38)}$.

En relación a las propiedades psicométricas del -GHQ-28- en población mexicana, ésta presentó una estructura similar a la versión original así como una confiabilidad y validez adecuada. Al ser un instrumento breve, fácil de aplicar y confiable para la práctica clínica y la investigación, resulta relevante para determinar la magnitud de la salud general de los cuidadores primarios.

Se ha encontrado que casi el $50 \%$ de los cuidadores reportan una deficiente calidad de $\mathrm{vida}^{(8)}$, un $60 \%$ alguna enfermedad crónica con un peso muy importante de problemas potencialmente invalidantes, que tienen un gran impacto en su vida cotidiana, al grado que se estima que el $20 \%$ de los cuidadores tienen dificultades para realizar su actividad normal para su edad, el $6 \%$ requieren de ayuda para realizar actividades de la vida diaria, y que se agrava en cuidadores mayores de 65 años que experimentan sobrecarga, y un riesgo de mortalidad $63 \%$ más elevado que la población de la misma edad que no es cuidador primario ${ }^{(39)}$; todo esto pone de manifiesto que las personas que cuidan la salud de otros de manera informal presentan con frecuencia una salud precaria ${ }^{(40)}$.

Por otro lado en la esfera psicológica el $32 \%$ de los cuidadores presentan síntomas de ansiedad, $22 \%$ de depresión, informan sentirse irritables y nerviosos $(23 \%)$ o tristes y agotados $(32 \%)^{(40,41)}$ estos síntomas se presentan con mayor riesgo cuando hay mayor dependencia física y deterioro cognitivo del paciente que cuidan, mayor tiempo que llevan cuidando a la persona y menor apoyo social percibido $^{(40)}$.

Estos hallazgos enfatizan la necesidad de evaluar y atender a los cuidadores primarios de una manera integral, y por ello el GHQ-28 representa una herramienta indispensable que ha sido probada en diferentes poblaciones: jóvenes ${ }^{(27)}$, pacientes de consulta externa ${ }^{(23)}$, y en población adulta ${ }^{(30)}$, y en todos ellos se ponen en evidencia estructuras factoriales similares.

La diferencia en la estructura factorial obtenida en el presente estudio y el GHQ-28 original posiblemente se deban a la demanda física y psicológica a la que está sometida esta población, y a los efectos directos en su salud las cuales son condiciones esperadas por las afectaciones descritas anteriormente.

Cabe resaltar que la importancia de evaluar e intervenir oportunamente en los cuidadores, no sólo los beneficia a ellos, sino que se ha documentado que el estado funcional del paciente correlaciona significativamente con dolor, vitalidad, función social, salud mental, bienestar y evaluación general de la salud del cuidador ${ }^{(42)}$.

\subsection{Implicaciones para la práctica y la investigación}

El GHQ-28 es un instrumento que ha demostrado un adecuado nivel de sensibilidad como instrumento de screening para detectar sujetos que requieren atención psiquiátrica, por lo que en el caso de los cuidadores primarios resulta una herramienta de gran utilidad debido a su facilidad de administración como un primer acercamiento que nos permita identificar con claridad a aquellos que requieren ser evaluados y atendidos por un servicio de psicooncología y/o psiquiatría; lo que representa un ahorro de recursos materiales, humanos y temporales en la evaluación 
de cuidadores primarios; y brinda la posibilidad de mejorar la atención integral del paciente oncológico y su familia lo cual es uno de los objetivos prioritarios de la psicooncología.

Como resultado de la validación del Cuestionario General de Salud de Goldberg se pueden proponer recomendaciones que ayudarán a mejorar las opciones de atención, que permitan aminorar el nivel de afectación a la salud asociada a este rol; y que tengan un impacto directo en la evolución del paciente oncológico. El -GHQ-28- representa un instrumento válido y confiable, práctico y económico al proporcionar una aproximación adecuada para la detección de posibles afectaciones en la salud general de esta población. Finalmente los datos apoyan la necesidad de incrementar los esfuerzos orientados a hacer más disponibles los servicios de atención psicooncológica en el grupo de cuidadores de pacientes oncológicos.

\subsection{Limitaciones del estudio}

Se recomienda estudios adicionales en población mexicana para identificar la estabilidad del GHQ-28 a través del tiempo.

\section{Referencias bibliográficas}

1. Organización Mundial de la Salud Nota descriptiva No. 297. 2015. [Acceso 1 enero de 2017]. Disponible en: http://www.who.int/mediacentre/factsheets/fs297/es/

2. Barrón BS, Alvarado S. Desgaste físico y emocional del cuidador primario en cáncer. Cancerología 2009;4.39-46. [Acceso 1 enero 2017]. Disponible en: http://www.incan.org. $\mathrm{mx} /$ revistaincan/elementos/documentosPortada/1257541295.pdf

3. Organización Panamericana de la Salud. (2007). Resumen: estrategia regional y plan de acción para un enfoque integrado sobre la prevención y el control de las enfermedades crónicas. Disponible en: http://www.paho.org/spanish/ad/dpc/nc/reg-stratcncds.

4. Organización Mundial de la Salud. En línea 2002. [Acceso 1 enero de 2017]. Disponible en: http://apps.who.int/iris/bitstream/10665/67275/1/WHO_NMH7CCL_02.1.pdf

5. Mancini J, Baumstarck-Barrau K, Simeoni MC, Grob JJ, Michel G, Tarpin C, Loundou AD, Lambert A, Clément A, Auquier P. Quality of life in a heterogeneous sample of caregivers of cancer patients: an in-depth interview study. Eur J Cancer Care 2011;20:48392. Doi: $10.1111 / j .1365-2354.2010 .01227 . x$

6. World Health Organization. WHO Fact Sheet "Caregiving". Geneva: WHO; 1999.

7. Galindo O, Benjet C, Cruz-Nieto MH, Rojas-Castillo E, Riveros-Rosas A, Meneses, et al. Psychometric properties of the Zarit Burden Interview in Mexican caregivers of cancer patients. Psychooncology 2015;24:612-5. Doi: 10.1002/pon.3686

8. López MJ, Orueta R, Gómez-Caro S, Sánchez A, Carmona de la Morena J, Alonso FJ. El rol de cuidador de personas dependientes y sus repercusiones sobre su calidad de vida y su salud. Rev Clin Med FAM 2009;2:332-9 Doi: 10.4321/S1699-695X2009000200004

9. Ruiz-Benítez MÁ, Coca MC. El pacto de silencio en los familiares de los pacientes oncológicos terminales. Psicooncología 2008;5:53-69.

10. Rohleder N, Marin TJ, Ma R. Miller, GE. Biological cost of caring for a cancer patient: Dysregulation of pro- and anti-inflammatory signaling pathways. J Clin Oncol 2009;27:290915. Doi: $10.1200 / J C O .2008 .18 .7435$. 
11. Lutgendorf SK, Laudenslager ML. Care of the caregiver: stress and dysregulation of inflammatory control in cancer caregivers. J Clin Oncol 2009;27:2894-5. Doi: 10.1200/ JCO.2009.22.1523

12. Shilling V, Matthews L, Jenkins V, Fallowfield L. Patient-reported outcome measures for cancer caregivers: a systematic review. Qual Life Res 2016; 25:1859-76. Doi: 10.1007/ s11136-016-1239-0

13. Cameron JI, Franche RL, Cheung AM, Stewart DE. Lifestyle interference and emotional distress in family caregivers of advanced cancer patients. Cancer 2002; 94:521-7. Doi: 10.1002/cncr.10212

14. Romito F, Goldzweig G, Cormio C, Hagedoorn M, Andersen BL. Informal caregiving for cancer patients. Cancer 2013;119(S11):2160-9. Doi: 10.1002/cncr.28057

15. Rhee YS, Yun YH, Park S, Shin DO, Lee KM, Yoo HJ, et al. Depression in family caregivers of cancer patients: The feeling of burden as a predictor of depression. J Clin Oncol 2008;26:5890-5. Doi:10.1200/ JCO.2007.15.3957

16. Fletcher BS, Paul SM, Dodd MJ, Schumacher K, West C, Cooper B, et al. Prevalence, severity, and impact of symptoms on female family caregivers of patients at the initiation of radiation therapy for prostate cancer. J Clin Oncol 2008;26:599- 605. Doi:10.1200/ JCO.2007.12.2838

17. Maltby KF, Sanderson CR, Lobb EA, Phillips JL. Sleep disturbances in caregivers of patients with advanced cancer: A systematic review. Palliat Support Care 2017;18:1-16. Doi: $10.1017 /$ S1478951516001024.

18. Prue G, Santin O, Porter S. Assessing the needs of informal caregivers to cancer survivors: a review of the instruments. Psychooncology 2015;24:121-9. Doi: 10.1002/pon.3609

19. Alfaro-Ramírez OI, Morales-Vigil T, Vázquez-Pineda F, Sánchez-Román S, Ramos-del Río B, Guevara-López U. Sobrecarga, ansiedad y depresión en cuidadores primarios de pacientes con dolor crónico y terminales, Revista médica del Instituto Mexicano del Seguro Social 2008;46:485-94.

20. Deeken JF, Taylor KL, Mangan P, Yabroff KR, Ingham JM. Care for caregivers: a review of self-report instrument developed to measure the buder, needs, and quality of life of informal caregivers. J Pain Symptom Manage 2003; 26:922-53. Doi:10.1016/S08853924(03)00327-0

21. Goldberg D. Manual del General Health Questionnaire. Windsor: NFER Publishing, 1978.

22. Retolaza A, Mostajo A, De la Rica JR, Diaz de Garramiola A, Pérez de Loza, Aramberri I, Márquez I. Validación del Cuestionario de Salud General de Goldberg (versión 28 ítems) en consultas de atención primaria. Rev Asoc Esp Neurops 1993;13:187-94.

23. Goldberg DP. Hillier VF. A scaled version of the General Health Questionnaire. Psychol Med 1979;9: 139-45. Doi: 10.1017/S0033291700021644

24. Lobo A, Pérez-Echeverría MJ, Artal J. Validity of the scaled version of the General Health Questionnaire (GHQ-28) in a Spanish population. Psychol Med 1986;16:135-40. Doi: 10.1017/S0033291700002579

25. Medina-Mora ME, Padilla GP, Campillo-Serrano C, Mas CC, Ezbán M, Caraveo J, Corona J. The factor structure of the GHQ: a scaled version for a hopital's general practice service in Mexico. Psychol Med 1983;13:355-61. Doi:10.1017/S0033291700050984

26. Schmitz N, Kruse J, Tress W. Psychometric properties of the General Health Questionnaire (GHQ-12) in a German primary care sample. Acta PsychiatrScand 1999;100:462-8. Doi: 10.1111/j.1600-0447.1999.tb10898.x 
27. Romero M. Medina-Mora ME. Validez de una versión del cuestionario general de Salud, para detectar psicopatología en estudiantes universitarios. Salud Mental 1987;10:90-7.

28. Wernek U, Goldberg DP, Yalcin I, Ustün BT. The stability of the factor structure of the General Health Questionnaire. Psychol Med 2000;30:823-9.

29. Jacob KS, Bhugra D, Mann AH. The validation of the 12-item General Health Questionnaire among ethnic Indian women living in the United Kingdom. Psychol Med 1997;27:1215-7.

30. García CRV. Manual para la utilización del cuestionario de salud general de Goldberg: Adaptación cubana. Rev Cubana Med Gen Integr 1999;15:88-97.

31. Beck AT, Ward CH, Mendelson M, Mock J, Erbaungh J. An inventory for measuring depression. Arch Gen Psychiatry 1961;4:53-63. Doi:10.1001/ archpsyc.1961.01710120031004

32. Jurado S, Villegas ME, Méndez L, Rodríguez F, Loperena V, Varela R. La estandarización del inventario de depresión de Beck para los residentes de la ciudad de México. Salud Mental 1998;21:26-31.

33. Vázquez OG, Castillo ER, Huertas LA, García AM, Ponce JLA, Manzanilla EO, et al. Guía de práctica clínica para la atención psico-oncológica del cuidador primario informal de pacientes con cáncer. Psicooncología 2015;12:87-104. Doi: 10.5209/rev_PSIC.2015. v12.n1.48906

34. Rossi FS, Zotti AM, Massara G, Nuvolone G. A comparative assessment of psychological and psychosocial characteristics of cancer patients and their caregivers. Psychooncology 2003;12:1-7. Doi: 10.1002/pon.626

35. Yee JL, Schulz R. Gender differences in psychiatric morbidity among family caregivers: a review and analysis. Gerontologist 2000;40:147-64.

36. García-Calvente MM, Mateo I, Gutiérrez P. Cuidados y cuidadores en el sistema informal de salud. Granada: Escuela Andaluza de Salud Pública e Instituto Andaluz de la Mujer. 1999.

37. Weitzner MA, McMillan SC, Jacobsen PB. Family caregiver quality of life: differences between curative and palliative cancer treatment settings. J Pain Symptom Manage 1999;17:418-28. Doi: 10.1016/S0885-3924(99)00014-7

38. Bevans M, Sternberg EM. Caregiving burden, stress, and health effects among family caregivers of adult cancer patients. JAMA 2012;307:398-403. Doi:10.1001/jama.2012.29

39. Schultz R, Beach SR. Caregiving as a risk factor for mortality: the caregiver health effects study. JAMA 1999;282:2215-29. Doi:10.1001/jama.282.23.2215

40. García-Calvente MM, Mateo-Rodríguez I, Moroto-Navarro G. El impacto del cuidador en la salud y la calidad de vida de las mujeres. Gac San 2004;18:83-92

41. García-Calvente MM, Mateo I, Gutiérrez P. Cuidados y cuidadores en el sistema informal de salud. Granada: Escuela Andaluza de Salud Pública e Instituto Andaluz de la Mujer, 1999.

42. Caquero-Urizar A, Segovia-Lagos P, Urritia-Urritia Ú, Miranda C, Navarro E. Impacto de la relación de ayuda de cuidadores primarios en la calidad de vida de pacientes con cáncer avanzado. Psicooncología 2013;10:95-108. Doi. 10.5209/rev_PSIC.2013.v10.41950 